\title{
Willingness of women with early estrogen receptor-positive breast cancer to take adjuvant CDK4/6 inhibitors
}

\author{
N.J. Lipton BA, ${ }^{*}$ J. Jesin $\mathrm{BSc}_{1}^{*}$ E. Warner $\mathrm{MD} \mathrm{MSc}_{1}{ }^{+}$X. Cao $\mathrm{PhD}_{1}^{\dagger}$ A. Kiss $\mathrm{PhD}_{1}^{\dagger}$ D. Desautels $\mathrm{MD}_{1}^{\neq \S}$ \\ and K.J. Jerzak MD MSc ${ }^{* \dagger}$
}

\begin{abstract}
Background The steady decline in breast cancer (вСa) mortality has come at the cost of increasingly toxic and expensive adjuvant therapies. Trials evaluating the addition of 2 or 3 years of cyclin-dependent kinase 4/6 (CDK4/6) inhibitors to adjuvant endocrine therapy (ЕT) are ongoing, but the willingness of patients to take such additional therapy is unknown.
\end{abstract}

Methods We surveyed 100 consecutive postmenopausal women with nonmetastatic estrogen receptor-positive BCa who had initiated adjuvant ET within the preceding 2 years. Participants were asked about perceived recurrence risk, BCa worry, and overall health. They were then asked about their willingness to accept 2 years of treatment with an additional oral drug that would reduce recurrence by $40 \%$ for a range of baseline recurrence risks in 2 hypothetical scenarios.

Results Mean age of the 99 evaluable participants was 61.7 years. In the scenario with no drug toxicity, $85 \%$ of respondents were likely to accept the new drug for a reduction in recurrence to $30 \%$ from $50 \%$, but only $49 \%$ would take the drug if risk was reduced to $3 \%$ from $5 \%$. In a scenario with drug-induced fatigue, the corresponding drug acceptance rates were $55 \%$ and $39 \%$ respectively. For the second scenario, BCa worry was correlated with increased willingness to take the drug, even for only a $2 \%$ absolute reduction in recurrence risk.

Conclusions The willingness of patients with estrogen receptor-positive BCa to take an adjuvant CDK4/6 inhibitor will greatly depend on the expected benefit and toxicities described to them as well as on worry about BCa recurrence.

Key Words Breast cancer, patient preferences, CDK4/6 inhibitors, adjuvant therapy, endocrine therapy

Curr Oncol. 2020 June:27(3)127-134

www.current-oncology.com

\section{INTRODUCTION}

Breast cancer (BCa) is the most commonly diagnosed cancer worldwide in women and the second leading cause of female cancer death in economically developed countries $^{1}$. Although distant metastatic disease is generally incurable, approximately $95 \%$ of BCas are diagnosed in the absence of detectable metastases ${ }^{2}$. Thus, strategies to reduce $\mathrm{BCa}$-related death predominately involve optimizing adjuvant therapies to prevent distant recurrence. With progressive improvements in adjuvant therapy, the 5-year survival rate in Canada is currently $90 \%{ }^{3}$.
Approximately $75 \%$ of $\mathrm{BCa}$ is estrogen receptor-positive (ER+) and does not overexpress HER2 (HER2-) ${ }^{4}$. For ER+ BCa, endocrine therapy (ET), generally prescribed for 5-10 years, is the most effective adjuvant treatment modality, reducing the relative risk of recurrence by more than $50 \%$ and mortality by more than $30 \%^{5}$. Chemotherapy further reduces recurrence, although to a lesser extent, for patients with high-risk clinical features or high genomic risk (for example, a high Recurrence Score). Despite optimal adjuvant systemic therapy, the long-term risk of distant recurrence for women with ER+, HER2- breast cancer ranges from $10 \%$ to $41 \%$, depending on the specific prognostic features of the 
tumour ${ }^{6}$. One potential strategy to further lower recurrence risk is to add a cyclin-dependant kinase (CDK4/6) inhibitor to standard adjuvant ET.

The efficacy of CDK4/6 inhibitors has already been established in the metastatic setting. Three pivotal studies have demonstrated that CDK4/6 inhibitors, when given in conjunction with a nonsteroidal aromatase inhibitor, improve progression-free survival in postmenopausal women with ER+, HER2-metastatic disease by approximately 10 months in the first-line metastatic setting, with hazard ratios ranging from 0.54 to $0.58^{7-9}$. Similar results were obtained in the MONALEESA-7 trial, in which premenopausal women on ovarian suppression were randomized to either ribociclib or placebo in addition to tamoxifen or a nonsteroidal aromatase inhibitor. Favourable survival data have also emerged to support the use of CDK4/ 6 inhibitors in both pre- and postmenopausal women with metastatic ER+ breast cancer ${ }^{10-12}$.

Currently, 3 CDK4/6 inhibitors-abemaciclib, palbociclib, ribociclib - have been approved by the U.S. Food and Drug Administration, Health Canada, and the European Medicines Agency for the treatment of metastatic BCa when given together with ET. In light of the efficacy of those drugs in metastatic disease, phase III trials are currently underway to determine their efficacy in the adjuvant setting for patients with a moderate-to-high risk of recurrence on standard therapy.

The PALLAS trial is examining whether 2 years of palbociclib concurrent with the first 2 years of standard adjuvant ET improves disease-free survival in early ER+, HER2- disease (see NCT02513394 at https://ClinicalTrials.gov/). The NATALEE (NCT03701334) and monarchE (NCT03155997) trials, studying ribociclib for 3 years and abemaciclib for 2 years respectively, have similar objectives and methodologies. Those trials are likely to show a significant reduction in recurrence rates - and possibly even mortality. However, the cost of a 2- or 3-year course of a CDK4/6 inhibitor is extraordinarily high. For example, 2 years of letrozole costs CA $\$ 926$. Adding palbociclib over that period would cost an additional CA $\$ 150,000$ for the drug alone ${ }^{13}$, which does not include the additional monetary costs of routine blood tests and more frequent clinic visits, nor the corresponding negative effect on patient quality of life and workplace productivity.

Although many studies have evaluated decision-making by patients with BCa about surgical treatment options and adjuvant chemotherapy ${ }^{14-17}$, the available data about their preferences for ET and other adjuvant therapies are very limited. In the present study, we used scenarios with varying rates of efficacy and toxicity for a combined regimen to explore the hypothetical willingness of postmenopausal patients with BCa, recently started on adjuvant ET, to add an oral drug with the characteristics of a CDK4/ 6 inhibitor to their treatment regimen. A secondary aim was to determine factors associated with the women's decisions.

\section{METHODS}

The study was approved by the research ethics board of Sunnybrook Health Sciences Centre.

Sequential postmenopausal women with nonmetastatic ER+, HER2- BCa, who had been initiated on adjuvant ET within the preceding 2 years and who were attending a medical oncology or radiation oncology outpatient clinic at Sunnybrook Odette Cancer Centre, were offered participation in the study. Recruitment was continued until a convenience sample of 100 participants was reached. Patients were eligible if they were actively taking either tamoxifen or an aromatase inhibitor. All patients had previously undergone lumpectomy or mastectomy, and might have received adjuvant radiation or chemotherapy. Women who were unable to provide informed consent or were not fluent in written English were excluded.

Basic demographic data, tumour pathology, and previous treatments for the participants were extracted from their electronic records. The number of women to whom chemotherapy or a bisphosphonate (or both) had been offered, and whether those women had agreed or declined to take the suggested treatments, was also recorded.

In the survey (Table I), patients were first asked about their perceived risk of their BCa recurring within 10 years (using a continuous linear scale ranging from 0 to 100), fear of BCa recurrence (worry), and perceived overall state of health. The latter two questions were assessed using 5-point Likert scales.

Patients were then asked about their willingness to accept an oral drug, in addition to their current ET, for the reduction of risk of $\mathrm{BCa}$ recurrence in two hypothetical scenarios. In the first scenario, no side effects were attributed to the additional drug, and in the second scenario, the drug was described as causing fatigue in $50 \%$ of the women taking it. In both scenarios, monthly blood tests and visits to the hospital were required. Within each scenario, the predicted risk of recurrence with standard ET alone (baseline recurrence risk) was varied. The new drug was assumed to provide a $40 \%$ relative risk reduction, based on the hazard ratios observed in the metastatic setting, independent of baseline recurrence risk ${ }^{18}$. For example, if the baseline risk of recurrence was $20 \%$, it would be reduced to $12 \%$ with combination therapy. Similarly, the additional drug would reduce a baseline recurrence risk of $5 \%$ to $3 \%$.

Responses about the respondent's willingness to take the additional drug in each hypothetical situation were collected on a 5-point Likert scale (very likely, likely, unsure, unlikely, and very unlikely). In both scenarios, to avoid any potential bias in the responses, neither the specific drug nor its class was revealed to the patients. The survey was pilot-tested with members of the lay public to ensure that the questions were easily understood. In addition, because the survey was administered in person in the cancer centre, patients had an opportunity to clarify any question.

The 15-year predicted risk of death from BCa was calculated for each patient using the PREDICT online prognostic tool (https://breast.predict.nhs.uk/), because that estimate was thought to correlate best with the patient's objective 10year risk of recurrence, which could then also be correlated with the patient's self-perceived 10 -year risk of recurrence. (The PREDICT tool does not calculate recurrence risk.)

Descriptive statistics, including means with standard deviation and medians, are reported for the continuous variables. For categorical variables, frequencies and percentages are presented. Correlations between willingness to take medication and selected variables were assessed using the Spearman correlation. Correlations between fear 
TABLE I Survey concerning patient preferences for oral adjuvant therapy in early hormone receptor-positive breast cancer

\section{Introduction}

This questionnaire is designed to assess your willingness to accept a new oral pill in combination with your standard hormonal (anti-estrogen) therapy to lower the risk of recurrence of your breast cancer. Currently, this new drug is being used only to treat metastatic breast cancer. Trials are underway to study this drug in early breast cancer, but its effectiveness has not yet been shown in this setting, and it is NOT part of standard care at this time.

\section{Objective}

We hope that this study will help us understand which patients might be willing to accept additional treatment for their breast cancer, despite potential side effects and increased need for clinic visits and blood tests.

We greatly appreciate you taking time to help with this research by participating in this survey. Completion of the survey is voluntary, and you may skip any question if you do not feel comfortable responding. Information collected is anonymous and CONFIDENTIAL.

Question 1

How likely do you think it is that your breast cancer will recur somewhere in your body within the next 10 years? Please mark an " $\mathrm{X}$ " on the scale, which ranges from 0 to $100 \%$ risk of breast cancer recurrence. For example, if you mark $50 \%$, it means you believe that your cancer is as likely to recur as it is to not recur. Please note that we're asking about the risk AFTER any treatments you have had such as chemotherapy, radiation, and/or your current hormonal therapy.

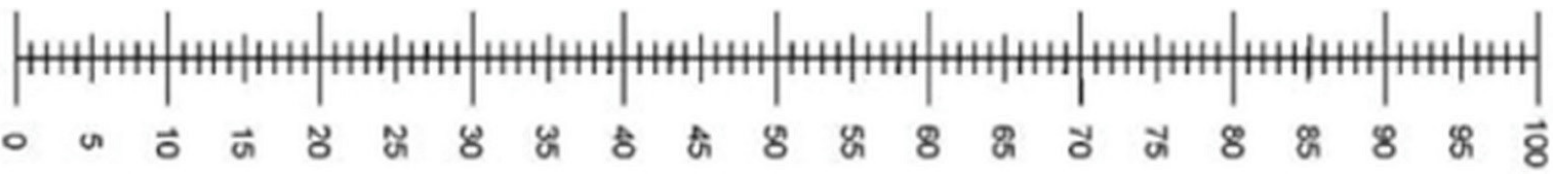

Question 2

How would you describe your overall state of health (please choose one)?
$\square$ Excellent
Very good
$\square$ Good
$\square$ Poor
Very poor

Question 3

How worried are you about breast cancer recurrence?

$\square$ Very worried $\square$ Worried $\square$ Unsure $\square$ Not very worried $\square$ Not at all worried

\section{Scenarios}

Please indicate your likelihood to accept a new oral pill in addition to hormone therapy for the following hypothetical scenarios.

Imagine that a new pill has been invented to treat early (non-metastatic) breast cancer in combination with your standard hormone therapy. The pill must be taken once a day for two years, and it would require a monthly blood test and visit to this hospital. Assume that the new pill does not have any side effects.

Scenario 1 - Willingness to take a new pill given varying risk of breast cancer recurrence

Imagine that your risk of breast cancer recurrence on or after standard hormone therapy is $50 \%$, and the new drug can further reduce that risk to $30 \%$. How likely are you to take this drug?

$$
\square \text { Very likely } \square \text { Likely } \square \text { Unsure } \square \text { Unlikely } \square \text { Very unlikely }
$$

Imagine that your risk of breast cancer recurrence on or after standard hormone therapy is $20 \%$, and the new drug can further reduce that risk to $12 \%$. How likely are you to take this drug?

$$
\square \text { Very likely } \square \text { Likely } \square \text { Unsure } \square \text { Unlikely } \square \text { Very unlikely }
$$

Assume that your risk of breast cancer recurrence on or after standard endocrine therapy is $10 \%$, and the new drug can further reduce that risk to $6 \%$. How likely are you to take this drug?

$$
\square \text { Very likely } \square \text { Likely } \square \text { Unsure } \square \text { Unlikely } \square \text { Very unlikely }
$$

Assume that your risk of breast cancer recurrence on or after standard endocrine therapy is $5 \%$, and the new drug can further reduce that risk to $3 \%$. How likely are you to take this drug?

$$
\square \text { Very likely } \square \text { Likely } \square \text { Unsure } \square \text { Unlikely } \square \text { Very unlikely }
$$

Scenario 2 - Willingness to take a new pill given varying side effects of the study drug

Assume that, in addition to monthly blood work and monthly visits, the drug also causes fatigue in approximately half of women. Without the new drug, no blood work is required, and visits are much less frequent.

How likely would you be to take this drug if your risk of breast cancer recurrence on or after standard hormone therapy is $50 \%$, and the new drug will further reduce that risk to $30 \%$.

$$
\square \text { Very likely } \square \text { Likely } \square \text { Unsure } \square \text { Unlikely } \square \text { Very unlikely }
$$

How likely would you be to take this drug if your risk of breast cancer recurrence on or after standard hormone therapy is $5 \%$, and the new drug will further reduce that risk to $3 \%$.

$$
\square \text { Very likely } \square \text { Likely } \square \text { Unsure } \square \text { Unlikely } \square \text { Very unlikely }
$$


of recurrence, age, self-perceived health, and worry about BCa recurrence were also examined using the Spearman correlation. Willingness of participants to take the additional adjuvant medication in the various scenarios of baseline recurrence risk or side effects was compared using the Wilcoxon signed-rank test. All statistical analyses were conducted using the SAS software application (version 9.4: SAS Institute, Cary, NC, U.S.A.).

\section{RESULTS}

Of 164 eligible women approached, 100 agreed to participate. The answers from 1 participant were ultimately excluded because of a demonstrated lack of comprehension of the scenarios. Of the 99 remaining participants, 62 had been taking an aromatase inhibitor (28 letrozole, 23 anastrozole, 10 exemestane, 1 unknown) and 37 had been taking tamoxifen for a mean duration of 13 months. Table II summarizes the baseline characteristics of the participants.

Of the participants, $18 \%$ described their overall state of health as excellent; $36 \%$, as very good; $40 \%$, as good; and $5 \%$, as poor. None described their health as very poor. Median self-perceived risk of breast cancer recurrence at 10 years was $10 \%$ (range: $0 \%-100 \%$ ). Based on the PREDICT model, the median 15-year risk of death from BCa was $5 \%$ (range: $1 \%-25 \%$ ). No significant correlation was found between self-perceived 10-year risk of recurrence on the part of the participants and objective predicted 15-year risk of death from $\mathrm{BCa}(p=0.06)$. Of the participants, $38 \%$ overestimated their absolute risk of recurrence by more than $10 \%$, with $25 \%$ of that group overestimating their risk by more than $25 \%$. Only 3 participants underestimated their recurrence risk by more than $10 \%$.

TABLE II Characteristics of the 99 study patients

\begin{tabular}{lc}
\hline \multicolumn{1}{c}{ Variable } & Value \\
\hline Age (years) & 61.7 \\
$\quad$ Mean & $34-90$ \\
$\quad$ Range & \\
Tumour size (cm) & 1.7 \\
$\quad$ Mean & $0.03-7.5$ \\
$\quad$ Range & 20 \\
\hline Lymph node-positive (\%) & \\
Tumour grade (\%) & 25 \\
1 & 61 \\
2 & 12 \\
3 & 1 \\
$\quad$ Missing & \\
\hline Surgery type (\%) & 37 \\
$\quad$ Mastectomy & 62 \\
Lumpectomy & \\
\hline Chemotherapy type (\%) & 17 \\
Adjuvant & 11 \\
Neoadjuvant & 77 \\
Adjuvant radiation (\%) & 46 \\
\hline Adjuvant bisphosphonate (\%) & \\
\hline
\end{tabular}

A small negative correlation between self-perceived overall health and perceived risk of recurrence was observed $(r=-0.26, p=0.01)$. Of the participants, $41 \%$ were not at all worried or not very worried about their risk of recurrence; $25 \%$ were unsure; and $33 \%$ were worried or very worried. A moderate correlation between perceived risk of recurrence and worry was observed $(r=0.34, p=$ $0.001)$. Increasing age was also negatively correlated with worry $(r=-0.24, p=0.01)$.

\section{Scenario 1}

The first clinical scenario probed the likelihood of patients accepting a new drug in combination with ET, for varying baseline risks of recurrence, in the absence of side effects, but with a requirement for monthly blood tests and hospital visits. As expected, the greater the absolute risk reduction, the more likely women were to accept the additional drug (Wilcoxon signed-rank test $p<0.0001$, Table III). The largest proportion of respondents (85\%) were likely or very likely to accept the new drug if the recurrence risk were to be reduced to $30 \%$ from $50 \%$, but if the recurrence risk were to be reduced to $3 \%$ from $5 \%$, only $49 \%$ indicated that they would be likely or very likely to accept the drug.

\section{Scenario 2}

The second scenario assessed the likelihood of participants accepting the new drug now with the potential side effect of fatigue reported by approximately $50 \%$ of users (still requiring monthly blood tests and visits to the hospital). If the hypothetical drug were to reduce the risk of BCa recurrence to $30 \%$ from $50 \%$, only $55 \%$ of respondents would be likely or very likely to accept the drug, and if the risk reduction were to be to $3 \%$ from $5 \%$, only $39 \%$ were still likely or very likely to accept it (Table IV). Willingness to take the drug was significantly greater for the 20 percentage point absolute reduction in recurrence than for the 2 percentage point benefit (Wilcoxon signed-rank test $p<0.0001$ ).

For the same absolute risk reduction of 20 percentage points, patients were significantly more likely to accept the drug in the absence of side effects than in the presence of a possibility of fatigue (Wilcoxon signed-rank test $p<0.0001$ ). The difference in the willingness of patients to take the drug, with or without the possibility of fatigue, was less when the absolute risk reduction was only 2 percentage points $(p=0.054)$.

Willingness to take the drug in either scenario was not observed to be correlated with age, self-perceived health, self-perceived risk of recurrence, or predicted 15-year risk of death from BCa. Although no correlation between worry and willingness to take the drug in the absence of side effects was evident, in the second scenario (possibility of fatigue as a side effect), greater worry was associated with an increased willingness to take the drug regardless of the degree of risk reduction (to $30 \%$ from $50 \%: r=0.22, p=0.03$; to $3 \%$ from $5 \%: r=0.25, p=0.01$ ).

Of the 62 women to whom a bisphosphonate was offered, 46 ( $74 \%$ ) chose to take it. Those 46 women were more likely than the 16 women who declined a bisphosphonate to accept the new drug in all scenarios, although the difference was only statistically significant in the scenarios that offered the largest absolute risk reduction without the risk 
TABLE III Likelihood that, in addition to endocrine therapy for early breast cancer, patients would accept a new oral drug with no side effects, but with monthly bloodwork and hospital visits

\begin{tabular}{|c|c|c|c|c|}
\hline \multirow[t]{2}{*}{ Response } & \multicolumn{4}{|c|}{ Likely to take the drug $(\%)$ for a reduction in the risk of breast cancer recurrence ... } \\
\hline & To $30 \%$ from $50 \%$ & To $12 \%$ from $20 \%$ & To $6 \%$ from $10 \%$ & To $3 \%$ from $5 \%$ \\
\hline Very likely & 50 & 44 & 34 & 27 \\
\hline Likely & 35 & 26 & 25 & 22 \\
\hline Unsure & 12 & 22 & 27 & 22 \\
\hline Unlikely & 3 & 6 & 10 & 22 \\
\hline Very unlikely & 0 & 2 & 4 & 7 \\
\hline
\end{tabular}

TABLE IV Likelihood that, in addition to endocrine therapy for early breast cancer, patients would accept a new oral drug with risk of fatigue and with the need for monthly bloodwork and hospital visits

\begin{tabular}{|c|c|c|}
\hline \multirow[t]{2}{*}{ Response } & \multicolumn{2}{|c|}{$\begin{array}{l}\text { Likely to take the drug }(\%) \text { for a reduction } \\
\text { in the risk of breast cancer recurrence ... }\end{array}$} \\
\hline & To $30 \%$ from $50 \%$ & To $3 \%$ from $5 \%$ \\
\hline Very likely & 33 & 18 \\
\hline Likely & 32 & 21 \\
\hline Unsure & 25 & 33 \\
\hline Unlikely & 7 & 19 \\
\hline Very unlikely & 3 & 9 \\
\hline
\end{tabular}

of fatigue (to $30 \%$ from $50 \%$ with no side effects: $p=0.01$; to $12 \%$ from $20 \%$, with no side effects: $p=0.04$ ). No significant correlation between cancer worry and willingness to take a bisphosphonate was evident ( $p=0.43$ ). Of 64 women for whom data about decision-making for chemotherapy were available ( 5 patients saw medical oncologists at other cancer centres), only 3 (4.7\%) declined a recommendation for chemotherapy.

\section{DISCUSSION}

In countries with a predominantly publicly funded health care system, decisions about whether to fund a particular drug must be based on strict criteria, including efficacy, toxicity, cost, and the size of the target population. In the near future, results of adjuvant studies randomizing women with ER+, HER2- breast cancer to standard ET with or without an adjuvant CDK4/ 6 inhibitor will be available. Based on the survival advantage now being reported for CDK4/6 inhibitors in the metastatic setting, those adjuvant studies will likely show a significant reduction in recurrence risk and, possibly, mortality. Although in those studies eligibility is restricted to patients with a moderate-to-high risk of BCa recurrence, there will undoubtedly be pressure on payers to fund the drugs even for women with a lower risk of recurrence. Funding decisions could depend in part on the number of eligible women who would actually take the drug. Even with standard ET, some women decline treatment upfront, with adherence among the rest dropping off progressively each year ${ }^{19}$.

In the present study, we aimed to estimate the percentage of postmenopausal women with ER+, HER2- breast cancer who would be willing to take a CDK4/6 inhibitor in addition to standard adjuvant ET. We also explored factors associated with their decision.

In our patient population, the median predicted 15 -year risk of $\mathrm{BCa}$ death was $5 \%$. The most relevant scenario presented to the patients was therefore the one in which risk of recurrence was reduced to $3 \%$ from $5 \%$ with mild fatigue in $50 \%$ of patients (plus a requirement for monthly bloodwork and hospital visits). In that scenario, only $39 \%$ of respondents indicated that they would be likely or very likely to accept the drug. Interestingly, willingness to take the drug with the chance of side effects in the hypothetical scenarios correlated with worry on the part of the participants about the risk of recurrence of their own BCa. It was surprising that $15 \%$ of women were unsure or unlikely to take the medication when the absolute risk reduction was marked (to $30 \%$ from $50 \%$ ) and no additional side effects were expected. The reasons for that observation were not explored, but the need for monthly bloodwork and additional visits to hospital was likely a factor. Ambivalent or negative attitudes toward preventive pharmacotherapy might also have contributed to the decision-making ${ }^{20}$. The fact that many participants who declined the additional drug in the scenarios with the greatest absolute benefit and the lowest risk of side effects had also declined a bisphosphonate suggests that a certain proportion of patients are reluctant to add any additional therapy to their standard adjuvant ET, regardless of the absolute benefit with concomitant drug tolerability.

Research into patient preferences generally outlines the views and values of patients about their treatment desires, and the issues that they deem important ${ }^{21}$. Preferences often depend on past experience and personal factors such as age and $\operatorname{sex}^{22}$. The wide variation in preferences for adjuvant systemic treatment among patients with BCa is clearly illustrated in a systematic review of fifteen studies, in which the median threshold for patients choosing adjuvant chemotherapy ranged from an absolute increase in survival of $0.1 \%$ to $10 \%$, or from 1 day to 5 years $^{23}$. The study reporting the lowest mean benefit might have been biased, because eligibility was restricted to patients who had received (and therefore agreed to) adjuvant chemotherapy, in many cases shortly before the interview ${ }^{24}$.

The findings in the present study support the importance of physicians recognizing the emotional factors that influence patient decision-making and ensuring that patients truly understand the objective benefits and risks of proposed treatments. However, our study, like others, 
shows that patient numeracy tends to be low and that many women vastly over- or underestimate their risk of recurrence $e^{25,26}$. Further research is necessary to determine how to present risk-benefit data to patients in a way that is truly comprehensible.

Interestingly, the greatest proportion of women in our study perceived themselves to be in excellent, very good, or good health. Further, only $6 \%$ were "very worried" and $27 \%$ were "worried" about breast cancer recurrence. That observation differs from a previous study in which $56 \%$ of 136 patients with BCa treated with curative intent experienced moderate-to-high levels of fear about disease recurrence, independent of the time since diagnosis. Fear of recurrence in that study, which included premenopausal women, declined significantly with increasing age ${ }^{27}$; hence, the difference in the degree of worry observed here might be explained by the exclusion of premenopausal women from our study and hence an older patient cohort.

Limitations of our study include the small sample size and a lack of data about the reasoning behind the participant responses. The latter compromise was made to limit the length of the questionnaire and to maximize participation. Additionally, participant decisions in the hypothetical scenarios might differ from their decisions in their personal case because, in the that situation, physicians often direct the discussion to influence patient decision-making, emphasizing certain risks or benefits depending on the physician's perception of what is best for the particular patient. Such differences are not surprising, nor necessarily problematic, because most patients want physician input when contemplating treatment choices ${ }^{28}$. In contrast, the scenarios in our study were presented in a completely neutral way, which likely explains the high proportion of participants who were unsure about whether they would take additional adjuvant therapy (33\%). A survey-based study by Fridman et al. ${ }^{29}$ demonstrated that physician "nudging" is considered ethical, acceptable, and even desirable by both clinicians and non-clinicians in decision-making about end-of-life care in oncology. However, a study of women with early-stage вСа suggested that communication of the oncologist's own treatment preferences is associated with more decisional conflict, particularly for older patients ${ }^{30}$.

Given that the science of decision-making is quite complex, with varying styles documented in the literature $^{31}$, a "one size fits all" approach to help patients make informed treatment decisions is not likely to be successful. A study of postmenopausal patients with BCa demonstrated significant variability in the amount of information and specific details requested when adjuvant ET is being contemplated $^{32}$, reinforcing that an individualized approach to decision-making is required.

An additional limitation of our study is that, for simplicity, the scenarios did not mention potential side effects of CDK4/6 inhibitors other than fatigue-for example, neutropenia, mucositis, and alopecia ${ }^{18}$. Fatigue was the only side effect mentioned in the survey because it is the most prevalent short- and long-term side effect of CDK4/ 6 inhibitors that adversely affects quality of life $\mathrm{e}^{33-35}$. Also, the health literacy and numeracy of the participants were not evaluated.

Finally, only postmenopausal patients were included because, at the time the study was planned, data about the efficacy of CDK4/6 inhibitors in premenopausal women were limited.

\section{CONCLUSIONS}

The progressive reduction in BCa mortality observed since the end of the $1980 \mathrm{~s}^{36}$ has resulted, for the most part, from an increasing intensity of adjuvant therapies. However, such increases in treatment intensity come at a significant cost to patient quality of life and a monetary cost to the health care system. Inhibitors of CDK4/6 will likely be the next addition to the already lengthy and side-effect-laden adjuvant systemic therapy for the population of patients with ER+, HER2- disease. It is important that funding decision-makers realize that the uptake of such drugs by eligible patients will be far below $100 \%$. Perhaps more importantly, as therapeutic regimens become increasingly complex, oncologists will have to ensure that patients truly understand the potential benefits and risks of each treatment component being offered.

\section{CONFLICT OF INTEREST DISCLOSURES}

We have read and understood Current Oncology's policy on disclosing conflicts of interest, and we declare the following interests: KJJ has served as a consultant or speaker for Apobiologix, Eisai, Genomic Health, Novartis, Purdue Pharma, Pfizer, and Roche; she has also received research support from AstraZeneca and Eli Lilly. The remaining authors have no conflicts of interest to disclose.

\section{AUTHOR AFFILIATIONS}

*Division of Medical Oncology, Sunnybrook Odette Cancer Centre, and ${ }^{\dagger}$ Sunnybrook Research Institute, Toronto, ON; ${ }^{\ddagger}$ CancerCare Manitoba and ${ }^{\S}$ Department of Medicine, University of Manitoba, Winnipeg, MB.

\section{REFERENCES}

1. Bray F, Ferlay J, Soerjomataram I, Siegel RL, Torre LA, Jemal A. Global cancer statistics 2018: GLOBOCAN estimates of incidence and mortality worldwide for 36 cancers in 185 countries. $C A$ Cancer J Clin 2018;68:394-424.

2. Canadian Cancer Statistics Advisory Committee. Canadian Cancer Statistics 2018. Toronto, ON: Canadian Cancer Society; 2018.

3. United States, Department of Health and Human Services, National Institutes of Health, National Cancer Institute (NCI), Surveillance, Epidemiology, and End Results Program. Cancer Stat Facts: Female Breast Cancer [Web page]. Bethesda, MD: NCI; n.d. [Current version available at: https://seer. cancer.gov/statfacts/html/breast.html; cited 15 August 2019]

4. Lin NU, Winer EP. Advances in adjuvant endocrine therapy for postmenopausal women. J Clin Oncol 2008;26:798-805.

5. Early Breast Cancer Trialists' Collaborative Group. Aromatase inhibitors versus tamoxifen in early breast cancer: patient-level meta-analysis of the randomised trials. Lancet 2015;386:1341-52.

6. Pan H, Gray R, Braybrooke J, et al. 20-Year risks of breastcancer recurrence after stopping endocrine therapy at 5 years. N Engl J Med 2017;377:1836-46.

7. Finn RS, Martin M, Rugo HS, et al. Palbociclib and letrozole in advanced breast cancer. $N$ Engl J Med 2016;375:1925-36.

8. Hortobagyi GN, Stemmer SM, Burris HA, et al. Ribociclib as first-line therapy for HR-positive, advanced breast cancer. N Engl J Med 2016;375:1738-48.

9. Goetz MP, Toi M, Campone M, et al. MONARCH 3: abemaciclib as initial therapy for advanced breast cancer. J Clin Oncol 2017;35:3638-46. 
10. Im SA, Lu YS, Bardia A, et al. Overall survival with ribociclib plus endocrine therapy in breast cancer. $N$ Engl J Med 2019;381:307-16.

11. Turner NC, Slamon DJ, Ro J, et al. Overall survival with palbociclib and fulvestrant in advanced breast cancer. $N$ EnglJ Med 2018;379:1926-36.

12. Sledge GW Jr, Toi M, Neven P, et al. The effect of abemaciclib plus fulvestrant on overall survival in hormone receptorpositive, ERBB2-negative breast cancer that progressed on endocrine therapy-MONARCH 2: a randomized clinical trial. JAMA Oncol 2019;6:116-24.

13. pan-Canadian Oncology Drug Review (pCODR).pan-Canadian Oncology Drug Review Final Economic Guidance Report: Palbociclib (Ibrance) for Advanced Breast Cancer-Resubmission. Toronto, ON: pCODR; 2016. [Available online at: https:// www.cadth.ca/sites/default/files/pcodr/pcodr_palbociclib _ibrance_resub_abc_fn_egr.pdf; cited 14 August 2019]

14. Lee WQ, Tan VKM, Choo HMC, et al. Factors influencing patient decision-making between simple mastectomy and surgical alternatives. BJS Open 2018;3:31-7.

15. Storm-Dickerson T, Das L, Gabriel A, Gitlin M, Farias J, Macarios D. What drives patient choice: preferences for approaches to surgical treatments for breast cancer beyond traditional clinical benchmarks. Plast Reconstr Surg Glob Open 2018;6:e1746.

16. Paraskeva N, Guest E, Lewis-Smith H, Harcourt D. Assessing the effectiveness of interventions to support patient decision making about breast reconstruction: a systematic review. Breast 2018;40:97-105.

17. Chao C, Studts JL, Abell T, et al. Adjuvant chemotherapy for breast cancer: how presentation of recurrence influences decision-making. J Clin Oncol 2003;21:4299-305.

18. Spring LM, Wander SA, Zangardi M, Bardia A. CDK 4/6 inhibitors in breast cancer: current controversies and future directions. Curr Oncol Rep 2019;21:25.

19. Hagen KB, Aas T, Kvaløy JT, Søiland H, Lindb R. Corrigendum to "Adherence to adjuvant endocrine therapy in postmenopausal breast cancer patients: a 5-year prospective study" [The Breast, 44, April 2019, 52-58]. Breast 2019;45:118.

20. Jerzak KJ, Pallan S, Gerstein HC. Willingness to take drugs to prevent serious chronic disease. J Diabetes 2013;6:76-80.

21. PEBC's Ovarian Oncology Guidelines Group. A systematic review of patient values, preferences and expectations for the treatment of recurrent ovarian cancer. Gynecol Oncol 2017;146:392-8.

22. Damm K, Vogel A, Prenzler A. Preferences of colorectal cancer patients for treatment and decision-making: a systematic literature review. Eur J Cancer Care (Engl) 2014;23:762-72.

23. Hamelinck VC, Bastiaannet E, Pieterse AH, et al. Patients' preferences for surgical and adjuvant systemic treatment in early breast cancer: a systematic review. Cancer Treat Rev 2014;40:1005-18.

24. Duric VM, Stockler MR, Heritier S, et al. Patients preferences for adjuvant chemotherapy in early breast cancer: what makes AC and CMF worthwhile now? Ann Oncol 2005;16:1786-94.

25. Hawley ST, Janz NK, Jagsi R, et al. Recurrence risk perception and quality of life after treatment of breast cancer [abstract 175]. J Clin Oncol 2016;34:. [Available online at: https:// ascopubs.org/doi/abs/10.1200/jco.2016.34.7_suppl.175; cited 1 June 2020]

26. Iacolo EK, Makari-Judson G, Mertens WC, et al. Perceived recurrence risk and health behavior change among breast cancer survivors. J Womens Health Gynecol 2014;1:1-8.

27. Marieke HJ, van den Beuken-van Everdingen, Peters ML, et al. Concerns of former breast cancer patients about disease recurrence: a validation and prevalence study. Psychooncology 2008;17:1137-45.

28. Tamirisa NP, Goodwin JS, Kandalam A, et al. Patient and physician views of shared decision making in cancer. Health Expect 2017;20:1248-53.

29. Fridman I, Hart JL, Yadav KN, Higgins ET. Perspectives on using decision-making nudges in physician-patient communications. PLoS One 2018;13:e202874.

30. Step MM, Siminoff LA, Rose JH. Differences in oncologist communication across age groups and contributions to adjuvant decision outcomes. J Am Geriatr Soc 2009; 57(suppl 2):S279-82.

31. Caldon LJM, Walters SJ, Reed MWR. Changing trends in the decision-making preferences of women with early breast cancer. Br J Surg 2008;95:312-18.

32. Feldman-Stewart D, Madarnas Y, Mates M, et al. Information for decision making by post-menopausal women with hormone receptor positive early-stage breast cancer considering adjuvant endocrine therapy. Breast 2013;22:919-25.

33. Mccabe RM, Grutsch JF, Braun DP, Nutakki SB. Fatigue as a driver of overall quality of life in cancer patients. PLoS One 2015;10:e0130023.

34. Hofman M, Ryan JL, Figueroa-Moseley CD, Jean-Pierre P, Morrow GR. Cancer-related fatigue: the scale of the problem. Oncologist 2007;12(suppl 1):4-10.

35. Dagnelie PC, Pijls-Johannesma MCG, Lambin P, Beijer S, De Ruysscher D, Kempen GIJM. Impact of fatigue on overall quality of life in lung and breast cancer patients selected for high-dose radiotherapy. Ann Oncol 2007;18:940-4.

36. Desantis CE, Ma J, Golding Sauer A, Newman LA, Jemal A. Breast cancer statistics, 2017, racial disparity in mortality by state. CA Cancer J Clin 2017;67:439-48. 